\title{
Inhibition of CXCR4 inhibits the proliferation and osteogenic potential of fibroblasts from ankylosing spondylitis via the $\mathbf{W n t} / \boldsymbol{\beta}$-catenin pathway
}

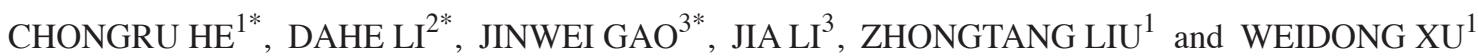 \\ ${ }^{1}$ Department of Orthopedics, Changhai Hospital Affiliated to The Second Military Medical University, \\ Shanghai 200433; ${ }^{2}$ Department of Orthopedics, The 960th Hospital of People's Liberation Army, Tai'an, Shandong 271000; \\ ${ }^{3}$ Department of Orthopedics, Jiangyan Traditional Chinese Medicine Hospital, Taizhou, Jiangsu 225500, P.R. China
}

Received March 4, 2018; Accepted October 15, 2018

DOI: $10.3892 / \mathrm{mmr} .2019 .9980$

\begin{abstract}
Ankylosing spondylitis (AS) is an autoimmune condition characterized by chronic inflammation and abnormal ossification as the primary features of the disease. The aim of the present study was to investigate the role of C-X-C chemokine receptor type 4 (CXCR4) in ossification from patients with AS. CXCR4 expression was assessed by western blot analysis and immunohistochemistry analysis of tissues obtained from patients with AS and controls. Fibroblasts were isolated, cultured and incubated with AMD 3100 and stromal cell-derived factor-1 to inhibit and promote CXCR4 levels, respectively. CXCR4 was upregulated in hip synovial tissues from patients with AS compared with that observed in controls. AS fibroblasts exhibited increased proliferation and growth rates. Inhibition of CXCR4 increased the phosphorylation of $\beta$-catenin and downregulated the expression of $\beta$-catenin, v-myc avian myelocytomatosis viral oncogene homolog, cyclin D1 and osteocalcin. Alizarin red staining demonstrated a decrease in biomineralization activity following the inhibition of CXCR4. These data support the hypothesis that inhibiting CXCR4 in patients with AS may suppress the ossification of fibroblasts.
\end{abstract}

Correspondence to: Professor Weidong $\mathrm{Xu}$, Department of Orthopedics, Changhai Hospital Affiliated to The Second Military Medical University, 168 Changhai Road, Yangpu, Shanghai 200433, P.R. China

E-mail: neuroletters@163.com

*Contributed equally

Abbreviations: AS, ankylosing spondylitis; CXCR4, chemokine receptor 4; SDF1, stromal cell-derived factor-1

Key words: ankylosing spondylitis, osteogenesis, fibroblasts, chemokine receptor 4 , proliferation

\section{Introduction}

Ankylosing spondylitis (AS), a common spondyloarthropathy, is an inflammatory rheumatic disease with a predilection for the axial skeleton. Clinical hallmarks of AS include sacroiliitis, uveitis, enthesitis and persistent spinal inflammation. The diagnosis of AS is usually difficult as the pathogenic mechanism of development and maintenance of the disease remains poorly understood $(1,2)$. However, it is considered to be immune-mediated and to have a marked genetic association with the class I human leukocyte antigen allotype HLA-B27 (3). Previous studies have suggested that sensations of hip stiffness are usually due to issues with the hip ligaments $(4,5)$, while the most common type of ligament tissue cells involved are fibroblasts. Fibroblasts derived from mesenchymal stem cells (MSC) with osteogenic properties are involved in ligament ectopic ossification, the origin of ligament ossification dynamic cells (6-9). However, the specific mechanisms involved in AS development have not been elucidated.

$\mathrm{C}-\mathrm{X}-\mathrm{C}$ chemokine receptor type 4 (CXCR4) is a member of the CXC chemokine factor family and stromal cell-derived factor-1 (SDF-1) the specific receptor which consists of an even transmembrane structure at the cell surface. The SDF-1/CXCR4 signaling axis serves an important role in several biological processes; it is involved in tumor cells, in particular cell transformation, migration and homing, in embryonic development, immune regulation, and is the primary regulator of tumorigenesis (10). In addition, the increase level of CXCR4 in human fetal MSCs was demonstrated to improve the success rate and the mechanical strength of bone allografts in rat models of osteogenesis. SDF-1 is a member of the CXC family, also known as C-X-C motif chemokine ligand 12 , and has been identified to be expressed by a number of different cell types (11-13). Previous studies have demonstrated the role of SDF-1 in the chemotaxis of stem cells, progenitor cells, and organ-specific homing through its interaction with CXCR4 $(14,15)$. Furthermore, the interaction between SDF-1 and CXCR4 may serve a crucial role during embryogenesis in cardiogenesis, hematopoiesis, vascular development and cerebellar development (16). Expression of the SDF-1/CXCR4 pathway in mature osteoblasts also results in feedback 
inhibition of osteoclast pool size, thereby affecting the homeostasis of bone formation and resorption (17). Expression of SDF1 was demonstrated to be induced in a mouse model of periosteum injury, through the SDF1/CXCR4 pathway during the recruitment of MSCs to the injury site within the bone cells involved in cartilage repair (18).

Therefore, in the present study, the differences in the expression levels of CXCR4 between AS and control hip synovial tissues was firstly examined, and whether blocking CXCR4 was able to alleviate osteogenesis was then explored. Subsequently, the function of differentially expressed proteins was investigated, in an attempt to clarify the pathogenesis of AS.

\section{Materials and methods}

Tissues samples and primary culture of hip capsule fibroblasts. The present study was approved by the Ethics Committee of Second Military Medical University (Shanghai, China). All samples were obtained from patients with AS (male:female $=11: 2, n=13$ ) and patients with aseptic necrosis of the femoral head (control; $n=8$ ). All of the patients underwent total hip arthroplasty at Changhai Hospital (Shanghai, China) between January 2017 and December 2017. Patients with AS were diagnosed according to the Modified New York Criteria (1984) (19). The age of patients with AS ranged from 8 to 20 years, with an mean age of 42.67 years. Ligaments were acquired from the femoral neck during surgery. None of the patients had taken anti-osteoporosis drugs, glucocorticoids, anti-tumor necrosis factor-drugs or NSAIDS at least 2 months prior to surgery. All patients agreed to participate and provided written informed consent. Fibroblasts were isolated from ligament tissues using the following method: The ligament tissue was washed twice with Dulbecco's modified Eagle's medium (DMEM; Thermo Fisher Scientific, Inc., Waltham, MA, USA) and finely minced. The minced tissue was incubated with $5 \mathrm{ml}$ DMEM containing $1 \mathrm{mg} / \mathrm{ml}$ collagenase type II (Sigma-Aldrich; Merck KGaA, Darmstadt, Germany) at $37^{\circ} \mathrm{C}$ for $6 \mathrm{~h}$, then with $5 \mathrm{ml} 0.25 \%$ trypsin at $37^{\circ} \mathrm{C}$ for $30 \mathrm{~min}$, filtered through a nylon mesh of $75 \mu \mathrm{m}$ and then washed three times. The single cell suspension was cultured in DMEM supplemented with $10 \%$ fetal bovine serum (FBS; Thermo Fisher Scientific, Inc.), penicillin (100 U/ml) and streptomycin $(100 \mu \mathrm{g} / \mathrm{ml})$ in a humidified $5 \% \mathrm{CO}_{2}$ atmosphere at $37^{\circ} \mathrm{C}$ overnight. Subsequently, non-adherent cells were removed, and adherent cells were re-cultured in DMEM plus $10 \%$ FBS. Confluent cells were trypsinized with $0.05 \%$ trypsin (Thermo Fisher Scientific, Inc.) and re-cultured. All ligament fibroblasts from the third passage were used for all subsequent experiments, as these cells were more purified compared with the first and second passages, and more similar to cells in vivo. Fibroblasts were identified by flow cytometry with CD90-FITC from GeneTex (GeneTex, Inc., Irvine, CA, USA) using a BD FACSCalibur flow cytometer (BD Biosciences, Franklin Lakes, NJ, USA).

Western blot analysis. Hip tissues in the AS and control groups were lysed in ice-cold lysis buffer [1\% Triton X-100, $20 \mathrm{mmol} / 1$ Tris-HCl (PH=8.0), $137 \mathrm{mmol} / \mathrm{l} \mathrm{NaCl}, 10 \%$ glycerol (v/v), 2 mmol/1 EDTA, $1 \mathrm{mmol} / 1$ phenylmethysulfonyl fluoride, $10 \mu \mathrm{g} / \mathrm{ml}$ leupeptin, $50 \mu \mathrm{g} / \mathrm{ml}$ trypsin inhibitor, $1 \mathrm{mmol} / 1$ sodium orthovanadate] for $15 \mathrm{~min}$. The amount of protein was determined using the BCA protein assay kit (Pierce; Thermo Fisher Scientific, Inc., Waltham, MA, USA). Protein $(100 \mu \mathrm{g})$ was loaded onto an $8 \%$ SDS-PAGE gel and transferred onto polyvinylidenedifluoride (PVDF) membranes (Bio-Rad Laboratories, Inc., Hercules, CA, USA). Membranes were blocked for $1 \mathrm{~h}$ at room temperature in 5\% skimmed milk, washed with TBST $(150 \mathrm{mM} \mathrm{NaCl}, 10 \mathrm{mM}$ Tris $\mathrm{pH} 7.4$, $0.1 \%$ Tween-20). Primary antibodies used included: Rabbit polyclonal CXCR4 (cat. no. sc-374159; 1:400; Santa Cruz Biotechnology, Inc., Dallas, TX, USA;), polyclonal $\beta$-catenin (cat. no. sc-316059; 1:1,000; Santa Cruz Biotechnology, Inc.), polyclonal p- $\beta$-catenin (cat. no. sc-316098; 1:500; Santa Cruz Biotechnology, Inc.), or polyclonal GAPDH (cat. no. ab8245; 1:500; Abcam, Cambridge, MA, USA). The antigen-antibody complexes were detected using peroxidase-labeled goat anti-rabbit antibodies (cat. no. ab95071; 1:800; Abcam) in TBS-T containing $2.5 \%$ non-fat dry milk for $1 \mathrm{~h}$ at room temperature. The results were visualized using the enhanced chemiluminescence Plus detection system (Abcam, Cambridge, MA, USA), and band areas/intensities for all proteins were measured using Image $\mathrm{J}$ software (version 1.8.0; National Institutes of Health, Bethesda, MD, USA).

Immunohistochemistry. All Immunohistochemistry (IHC) analyses was performed on formalin-fixed and paraffin-embedded samples. Paraffin blocks were sectioned to 4- $\mu \mathrm{m}$ thickness. Then, poly-L-lysine-coated slides were used to promote adhesion of the paraffin-section to the slides. The sections were dewaxed, rehydrated, and antigen-repaired. For dewaxing, the sections were baked in an incubator at $60^{\circ} \mathrm{C}$ for $2 \mathrm{~h}$, followed by cooling to room temperature and soaking in xylene for 10 min 3 times. For rehydrating, the sections were put into ethanol (I) for $5 \mathrm{~min}$, ethanol (II) for $5 \mathrm{~min}, 95 \%$ ethanol solution for $5 \mathrm{~min}, 80 \%$ ethanol solution for $5 \mathrm{~min}$, $70 \%$ ethanol solution for $5 \mathrm{~min}, 50 \%$ ethanol solution for $5 \mathrm{~min}$, and $\mathrm{dd}_{2} \mathrm{O}$ for 5 min successively. The sectioned tissue slides were incubated with the primary polyclonal rabbit antibodies: Polyclonal rabbit CXCR4 (cat. no. sc-374159; 1:400; Santa Cruz Biotechnology, Inc.) for $18 \mathrm{~h}$ at $4^{\circ} \mathrm{C}$ in a humidity chamber. Biotinylated secondary antibody anti-rabbit IgG (cat. no. ab80948; 1:2,000, Abcam) was incubated with the sections for $30 \mathrm{~min}$ at $37^{\circ} \mathrm{C}$, then incubated with extra-avidin peroxidase for $30 \mathrm{~min}$ at $37^{\circ} \mathrm{C}$. Images were captured under Nikon Eclipse (E600) fluorescent microscope (Nikon Corporation, Tokyo, Japan) at magnification $\mathrm{x} 400$, and then analyzed using Image-Pro Plus software (version 4.5.1; Media Cybernetics, Rockville, MD, USA).

Fibroblast verification. Flow cytometry was used for fibroblast verification. The CD90 marker was used to identify fibroblasts, as described previously (20-22). In brief, third-generation fibroblasts were collected, and a suspension with a density of $1 \times 10^{5}$ cells $/ \mathrm{ml}$ was prepared. Next, $12 \mathrm{ml}$ trypsin/EDTA solution was added to the $75 \mathrm{~cm}^{3}$ cell tubes with $1 \times 10^{6}$ cells $/ \mathrm{ml}$ in each tube. A total of $20 \mu 1$ fluorescein isothiocyanate-labeled mouse anti-human CD90 monoclonal antibody (cat. no. 11-0903-82; 1:1,000; Thermo Fisher Scientific, Inc.) was added, followed by incubation 
on ice for $20 \mathrm{~min}$ in the dark. Tubes without antibodies were used as the negative control. PBS (2 ml) was added to each tube and centrifuged at $400 \mathrm{x} \mathrm{g}$ for $6 \mathrm{~min}$ at $25^{\circ} \mathrm{C}$. Cells were washed twice with $0.5 \mathrm{ml}$ PBS. Flow cytometry was performed using BD FACSCalibur version 4.1 (BD Biosciences). Fibroblasts were plated into chamber slides and fixed with $3 \%$ formaldehyde, and then blocked with $5 \%$ BSA and incubated with anti-Vimentin (cat. no. ab20346; 1:2,000; Abcam,) overnight at $4^{\circ} \mathrm{C}$. Following incubation with the primary antibodies, cells were washed three times in PBS followed by $60 \mathrm{~min}$ of incubation at room temperature with anti-rabbit FITC (cat. no. Sfk12258; 1:1,000; Shanghai Shifeng Biological Technology, Co., Ltd., Shanghai, China,). Fluorescence was analyzed using the fluorescence microscopy (Nikon Corporation).

Cell proliferation assay. Cell proliferation was assessed using an MTS assay. Aliquots $(10 \mu 1)$ of MTS at a concentration of $0.1 \mathrm{mg} / \mathrm{ml}$ from the MTS cell proliferation assay kit (Promega Corporation, Madison, WI, USA) were added to the cell $\left(1 \times 10^{4}\right)$ cultures. After $4 \mathrm{~h}$ at $37^{\circ} \mathrm{C}$, the absorbance was measured at $490 \mathrm{~nm}$ with a spectrophotometer.

Osteogenesis induction. The fibroblasts were digested with $0.25 \%$ trypsin and cultured at $37^{\circ} \mathrm{C}$ in a humidified atmosphere containing $5 \% \mathrm{CO}_{2}$. Following attachment of the cells to the surface of the wells, osteogenic induction medium (OM) containing DMEM/F12 with $100 \mathrm{nM}$ dexamethasone, $50 \mathrm{mg} / \mathrm{l}$ ascorbic acid, $10 \mathrm{mM} \beta$-sodium glycerophosphate and $10 \%$ FBS was added. The medium contained mycillin and was changed every 2-3 days.

Cell proliferation assay following incubation with AMD 3100 or SDF-1, and osteogenesis induction. Following the induction of osteogenesis, AMD $3100(10 \mu \mathrm{M})$ or SDF-1 $(10 \mathrm{ng} / \mathrm{ml})$ was added to the fibroblasts. Cell proliferation was then assessed using the MTS assay as aforementioned.

Small interference RNA (siRNA) transfection assay. For in vitro knockdown studies, siRNA targeting rat CXCR4 (Invitrogen; Thermo Fisher Scientific, Inc.) were used. Control cultures were transfected with scramble siRNA (Invitrogen; Thermo Fisher Scientific, Inc.). Fibroblasts were seeded in a $6-\mathrm{cm}$ dish at a density of $5 \times 10^{5}$ cells/dish and prepared for transfection with $1 \mu \mathrm{g}$ CXCR4 siRNA or control siRNA. siRNA was added to Opti-MEM with Lipofectamine ${ }^{\circledR} 2000$ RNAi MAX (Invitrogen; Thermo Fisher Scientific, Inc.) for transfection according to the manufacturer's protocol. A total of $8 \mathrm{~h}$ following incubation, the medium was changed into fresh EBM-2 medium containing 15\% FBS. Cells were harvested at $48 \mathrm{~h}$ following transfection of siRNA. The sequences of the CXCR4 siRNA used were as follows: 5'-GACUGGUAC UUUGGGAAAUTT-3' and 3'-AUUUCCCAAAGUACC AGUCTT-5'. The scrambled sequence was 5'-GUAGCAGGG CAUGUAUUUATT-3' and 3'-UAAAUACAUGCCCUGCUA CTT-5', and was designed as a negative control. The efficiencies of CXCR4 siRNA and control siRNA were monitored by western blot analysis, as aforementioned. The fibroblasts were divided into two groups: i) The AS+OM group, containing osteogenesis-induced fibroblasts; and ii) AS+OM + si-CXCR4 group, containing osteogenesis-induced fibroblasts treated with si-CXCR4.

Western blot analysis following incubation with AMD 3100 or SDF-1, and osteogenesis induction. The cells were washed with ice-cold PBS and lysates were prepared in radioimmunoprecipitation assay lysis buffer $[50 \mathrm{mM}$ Tris- $\mathrm{Cl} \mathrm{pH}$ 7.4, $150 \mathrm{mM} \mathrm{NaCl}, 0.25 \%$ sodium deoxycholate, $1 \%$ NP-40, $100 \mathrm{mg} / \mathrm{ml}$ PMSF Protease Inhibitor Cocktail (Roche Diagnostics, Basel, Switzerland)]. Lysates were passed through a $1 \mathrm{ml}$ needle syringe to facilitate the disruption of the cell membranes and centrifuged at $10,000 \mathrm{x} \mathrm{g}$ for $15 \mathrm{~min}$ at $4^{\circ} \mathrm{C}$, and supernatants were collected. Protein concentrations of lysates were determined by PierceH BCA Protein Assay kit (Pierce; Thermo Fisher Scientific, Inc.). Protein $(100 \mu \mathrm{g})$ was loaded onto a $10 \%$ SDS-PAGE gel and transferred onto polyvinylidene difluoride membranes (Merck KGaA). Membranes were then blocked for $1 \mathrm{~h}$ at room temperature in 5\% skim milk, washed with TBST (150 mM NaCl, 10 mM Tris pH 7.4, 0.1\% Tween-20). Primary antibodies used included CXCR4 (cat. no. sc-374159; 1:800; Santa Cruz Biotechnology, Inc.), $\beta$-catenin (cat. no. sc-7963; 1:1,000; Santa Cruz Biotechnology, Inc.;), phosphorylated (p)- $\beta$-catenin (cat. no. sc-316059; 1:700; Santa Cruz Biotechnology, Inc.), glycogen synthase kinase $3 b$ (cat. no., sc-11757; GSK3b; 1:500; Santa Cruz Biotechnology, Inc.), frizzled-7 (FZD7; cat. no. sc-31061; 1:500; Santa Cruz Biotechnology, Inc.), alkaline phosphatase (ALP; cat. no., sc-214129; 1:1,000; Santa Cruz Biotechnology, Inc.), osteocalcin (OCN; cat. no., sc-139134; 1:1,500; Santa Cruz Biotechnology, Inc.), v-myc avian myelocytomatosis viral oncogene homolog (c-myc; cat. no., sc-261714; 1:1,000; Santa Cruz Biotechnology, Inc.) and cyclin-D1 (cat. no., sc-426371; 1:1,000; Santa Cruz Biotechnology, Inc.). Membranes were washed three times in TBST, incubated with horseradish peroxidase-conjugated secondary antibody (HRP; ab6721; 1:1,000; Abcam) at 1:5,000 in 5\% skim milk for $1 \mathrm{~h}$ at room temperature, washed and then processed with ECL Plus Western Blotting detection kit (Amersham Biosciences; GE Healthcare, Chicago, IL, USA). The filter was then incubated with the substrate and exposed to X-ray films (Thermo Fisher Scientific, Inc.), and analyzed using Image $\mathrm{J}$ software (version 1.8.0; National Institutes of Health, Bethesda, MD, USA).

Alizarin red staining. As described previously (23), cultured cells were stained with $0.1 \%$ alizarin red (Thermo Fisher Scientific, Inc.) for $30 \mathrm{~min}$ at $37^{\circ} \mathrm{C}$. Each well washed twice with PBS, then fixed in $10 \%$ formalin for $15 \mathrm{~min}$ at room temperature. Then, the wells were rinsed with distilled water, and $1 \mathrm{ml}$ alizarin red $\mathrm{S}$ was added to each well $(40 \mathrm{mM}$; $\mathrm{pH}$ 4.1). The 24-well plate was then placed on a shaker and incubated at room temperature for $20 \mathrm{~min}$. Then, $4 \mathrm{ml}$ distilled water was added to each well and rinsed for $5 \mathrm{~min}$, and this step was repeated 4 times.

Statistical analysis. Statistical analysis was performed using SPSS version 18.0 software (SPSS Inc., Chicago, IL, USA). The data are presented as mean \pm standard error of the mean, and differences between groups were analyzed by two way analysis of variance. Post-hoc pairwise comparisons were 
Table I. Basic clinical data for patients with AS.

\begin{tabular}{|c|c|c|c|c|c|}
\hline Patient no. & Age, y & Sex, M/F & Duration of disease, $y$ & HLA-B27 & NSAIDs used \\
\hline 1 & 29 & M & 8 & + & No \\
\hline 2 & 30 & M & 10 & + & No \\
\hline 3 & 27 & M & 7 & + & No \\
\hline 4 & 30 & M & 8 & + & No \\
\hline 5 & 33 & M & 6 & + & No \\
\hline 6 & 28 & M & 8 & + & No \\
\hline 7 & 33 & M & 7 & + & No \\
\hline 8 & 29 & M & 9 & + & No \\
\hline 9 & 52 & M & 30 & + & No \\
\hline 10 & 56 & M & 23 & + & No \\
\hline 11 & 54 & $\mathrm{~F}$ & 20 & + & No \\
\hline 12 & 77 & $\mathrm{~F}$ & 33 & + & No \\
\hline 13 & 65 & M & 31 & + & No \\
\hline
\end{tabular}

AS, ankylosing spondylitis; HLA-B27, human leukocyte antigen B27; NSAIDs, non-steroidal anti-inflammatory drugs.

performed using the Bonferroni test. $\mathrm{P}<0.05$ was considered to indicate a statistically significant difference.

\section{Results}

Upregulation of CXCR4 in AS ligament tissue. The basic clinical data of the two groups are summarized in Tables I and II; there were no significant differences between the two groups in terms of sex and age. The expression of CXCR4 in AS and control ligament tissues was then investigated. Immunohistochemistry (Fig. 1A and B) and western blot analysis (Fig. 1C and D) indicated that the expression of CXCR4 was upregulated in AS tissues compared to control tissues $(\mathrm{P}<0.01)$.

Upregulation of CXCR4 in AS fibroblasts. Primary fibroblasts were successfully cultured. Following cell sorting for fibroblasts, fibroblasts morphology was determined by vimentin immunocytochemistry (Fig. 2). To determine whether the fibroblasts in patients with AS were different from those in the control group, cell proliferation assays in the two fibroblasts groups were conducted. It was identified that AS fibroblasts exhibited an increased growth rate compared with the control group, as determined by the growth curve.

Blocking of CXCR4 inhibits fibroblast proliferation and osteogenic ability. The role of CXCR4 in the proliferation and osteogenesis of AS fibroblasts was examined by inhibiting CXCR4. Flow cytometric analysis indicated that the inhibition of CXCR4 through the addition of AMD 3100 led to a decrease in the proliferation of AS fibroblasts (Fig. 3). However, the proliferation of AS fibroblasts was significantly increased following the addition of SDF-1 (Fig. 3). Therefore, these data suggest that CXCR4 is involved in the proliferation of AS fibroblasts.

Next, it was identified that the AMD 3100 significantly suppressed the expression of CXCR4, while SDF-1 increased
Table II. Basic clinical data for the control group.

\begin{tabular}{lccc}
\hline Patient no. & Age, $y$ & Sex, M/F & Diagnosis \\
\hline 1 & 54 & M & NFH \\
2 & 61 & M & NFH \\
3 & 58 & F & NFH \\
4 & 27 & M & NFH \\
5 & 48 & M & NFH \\
6 & 49 & M & NFH \\
7 & 52 & F & NFH \\
8 & 44 & M & NFH \\
\hline
\end{tabular}

NFH, necrosis of the femoral head.

the level of CXCR4 in fibroblasts. Then, the downregulation of $\beta$-catenin, GSK3b, FZD7, c-myc, cyclin D1 and the osteogenesis markers ALP and OCN, and the upregulation of $p-\beta$-catenin were observed in fibroblasts following the blocking of CXCR4 using AMD 3100 (Fig. 4). Conversely, SDF-1 in fibroblasts led to the opposite results.

Alizarin red staining. Alizarin red staining for calcium revealed significantly decreased levels of mineralization following the addition of AMD 3100 (Fig. 5). By contrast, the amount of mineralization indicated a distinctive increasing trend following the addition of SDF-1 compared with that observed in the AS+OM and AS+OM+AMD 3100 groups.

CXCR4 promotes osteogenic and mineralization abilities through the Wnt pathway. Alizarin red staining was performed to investigate the mineralization ability of CXCR4. As indicated in Fig. 6A and B, the level of mineralization was markedly decreased following preincubation with CXCR4 siRNA $(\mathrm{P}<0.05)$. It was then identified that the si-CXCR4 significantly 

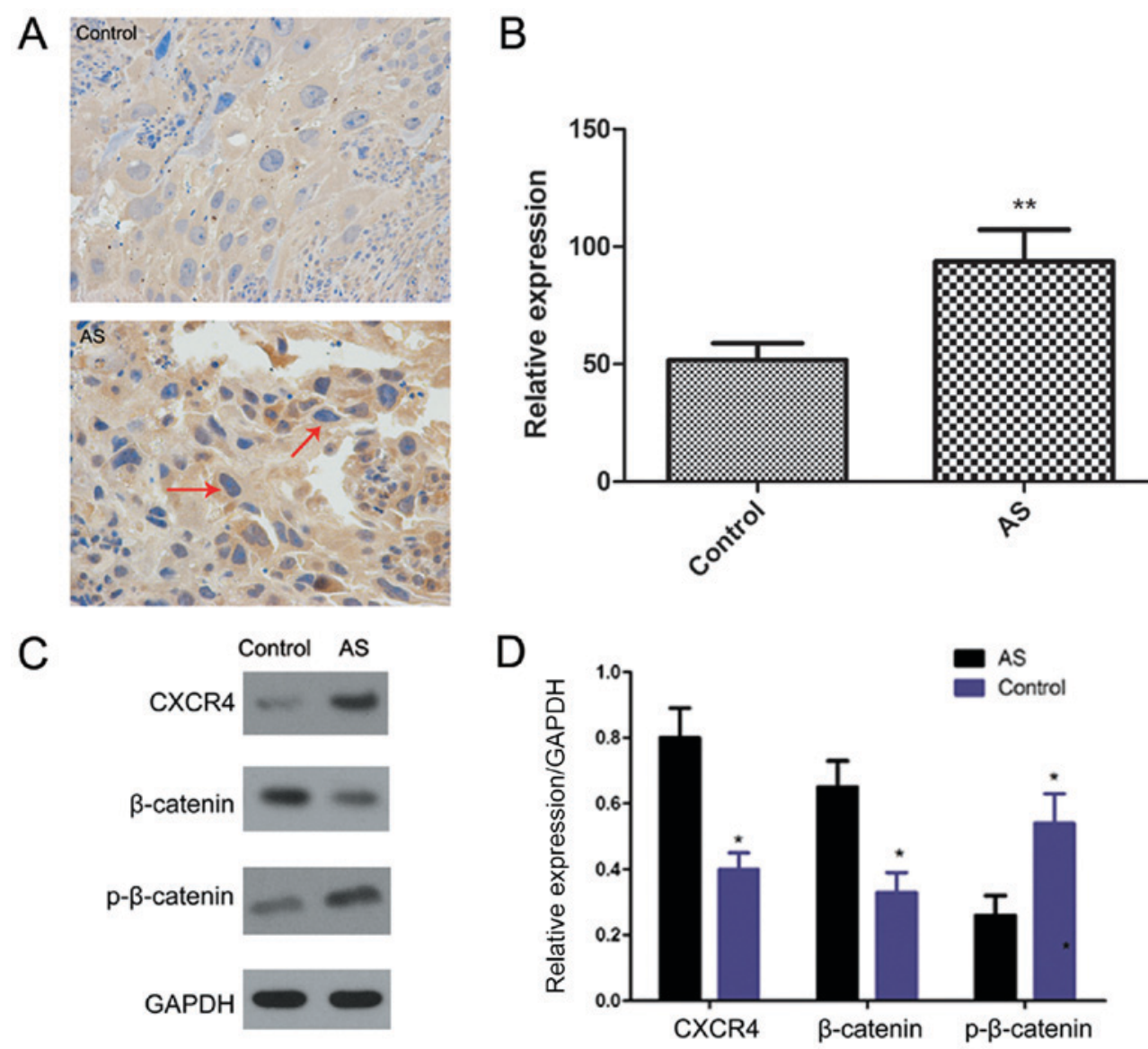

Figure 1. CXCR4, $\beta$-catenin, and p- $\beta$-catenin analysis in AS and control groups. (A) Immunohistochemical analysis of CXCR4 expression in hip synovial specimens obtained from patients from the control and AS groups (magnification, x100). (B) Quantitative analysis of immunohistochemical CXCR4 expression. The results are representative of three independent experiments with 10 samples each $\left({ }^{* *} \mathrm{P}<0.05\right.$ vs. control group). (C) Western blot analyses of the expression levels of CXCR4, $\beta$-catenin, and p- $\beta$-catenin in AS and control hip synovial tissues. (D) The bar graph demonstrates the result of the densitometric quantification using Image $\mathrm{J}$ software ( ${ }^{*} \mathrm{P}<0.05$ vs. AS group). The data represent the mean value from three independent experiments. $\mathrm{CXCR} 4$, $\mathrm{C}-\mathrm{X}-\mathrm{C}$ chemokine receptor type 4; p, phosphorylated; AS, ankylosing spondylitis.

suppressed the expression of CXCR4, while SDF-1 increased the level of CXCR4 in fibroblasts (Fig. 6C and D). Then, the downregulation of $\beta$-catenin, GSK3b, FZD7, c-myc, cyclin D1 and the osteogenesis markers ALP and OCN, and upregulation of phosphorylated $\beta$-catenin were observed in fibroblasts following the silencing of CXCR4. Collectively, these data suggested that CXCR4 promoted the functions of osteogenesis and mineralization formation in cultured fibroblasts.

\section{Discussion}

In the present study, the role of CXCR4 in AS fibroblasts proliferation and osteogenesis was examined. To the best of our knowledge, the present study demonstrated for the first time that upregulation of CXCR4 led to fibroblast proliferation and osteogenesis, while CXCR4 inhibition resulted in the opposite effect, and for the first time demonstrated an increase in CXCR4 levels in tissues obtained from patients with AS.

AS is am autoimmune disease characterized by chronic inflammation and abnormal ossification. At present, understanding of its pathogenesis is limited, and therefore the design of targeted drugs has not been available. The high morbidity rates of AS are a common cause of the burden to families and society (24). Musculoskeletal enthesitis is one of the primary features of the pathogenesis of AS, which involves ligaments in the joint capsule connected to the bone. Long-term follow-up imaging studies have demonstrated that these areas will eventually exhibit ossification (25). A previous study also demonstrated that in patients with stiff hip ligament ossification, ligament fibroblasts have been involved (9). Concomitantly, other studies have indicated that AS ligament fibroblasts exhibit a tendency towards ossification $(26,27)$.

Fibroblast cells are associated with joint remodeling, which occurs in rheumatic diseases including AS. Cluster of differentiation 90 (CD90) is a cell adhesion molecule and the smallest member of the immunoglobulin superfamily, with a molecular weight of 25-35 KDa; it has been demonstrated to be enriched in fibroblasts (28). CD90 positivity in fibroblast, when measured, has been revealed to be due to fibroblast contamination of the other cells, which has also been confirmed visually (29). Previous studies have indicated that different cell types may be identified based upon CD90 expression $(30,31)$. There was a loose correlation between CD90 expression and cuboidal morphology of non-activated cells (20). An additional commonly used cell marker for fibroblasts is vimentin, which has a high level of sensitivity for fibroblast labelling (32). Vimentin was originally used as an endothelial cell marker. Although vimentin may positively 

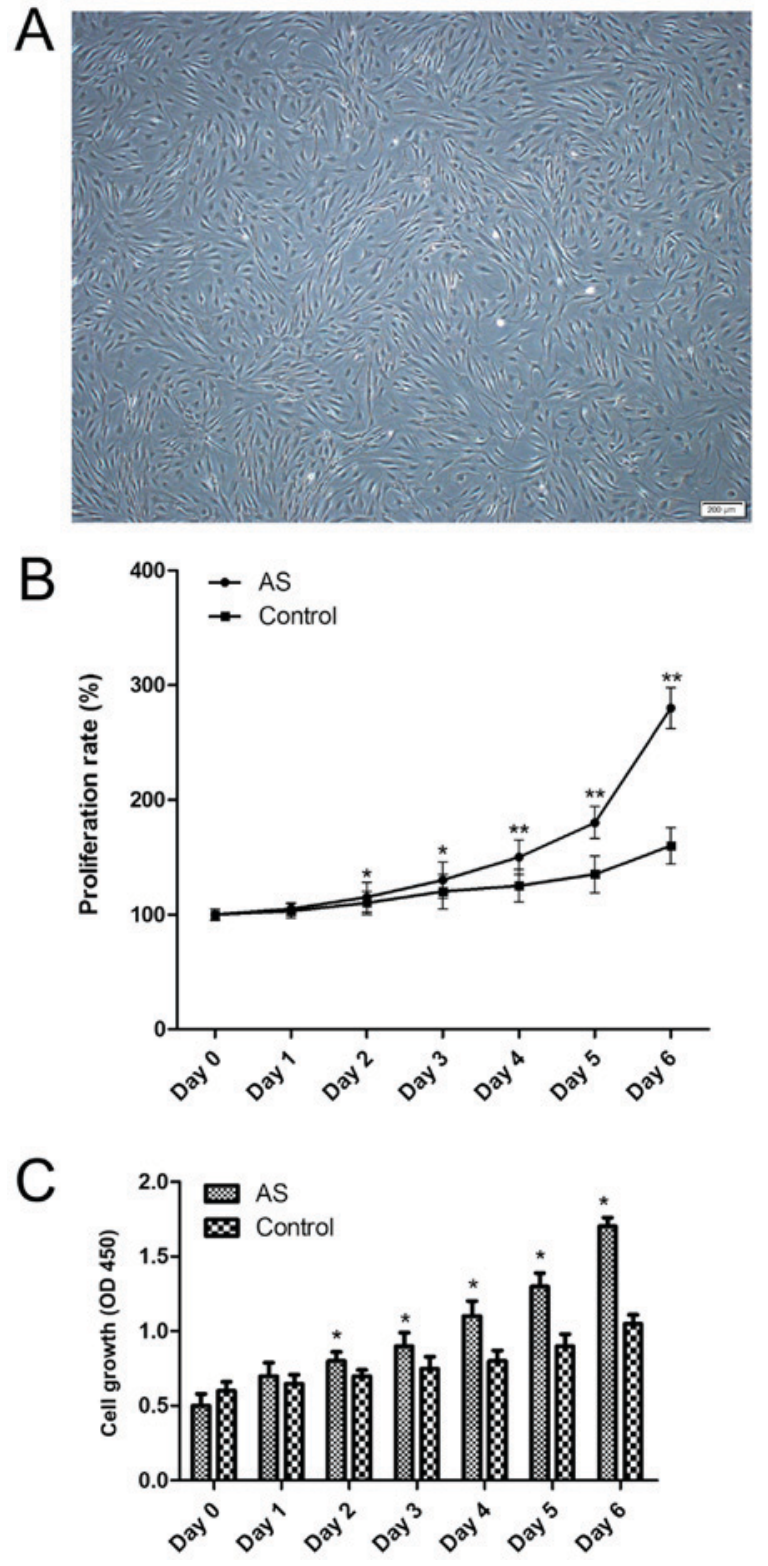

Figure 2. Growth evaluation of fibroblasts in AS and control groups. (A) Primary fibroblasts were successfully cultured and sub-cultured using the explant culture method (third generation). (B) Cell proliferation and (C) cell growth were measured using an MTS assay. This experiment was repeated three times. Error bars represent the standard deviation. ${ }^{*} \mathrm{P}<0.05$ and ${ }^{* *} \mathrm{P}<0.01$ vs. control. AS, ankylosing spondylitis.

identify fibroblasts, it also positively identifies macrophages and endothelial cells. In the present study, CD90 was used as the sole marker for fibroblasts identification, which may present a limitation. Additional experiments, including the use CD90 and vimentin as markers for fibroblasts, will be performed in future studies.

Previous studies have suggested that the SDF1-CXCR4 signaling axis is involved in a number of biological processes in vivo $(10,33)$. In the present study, the experimental data indicated that the expression level of CXCR4 was upregulated in the AS group compared with controls, indicating AS was triggered in fibroblasts. It was also identified that the AS group exhibited an increased level of expression of $\beta$-catenin compared with the control group, which is similar to the results from a previous study (34), suggesting that upregulated
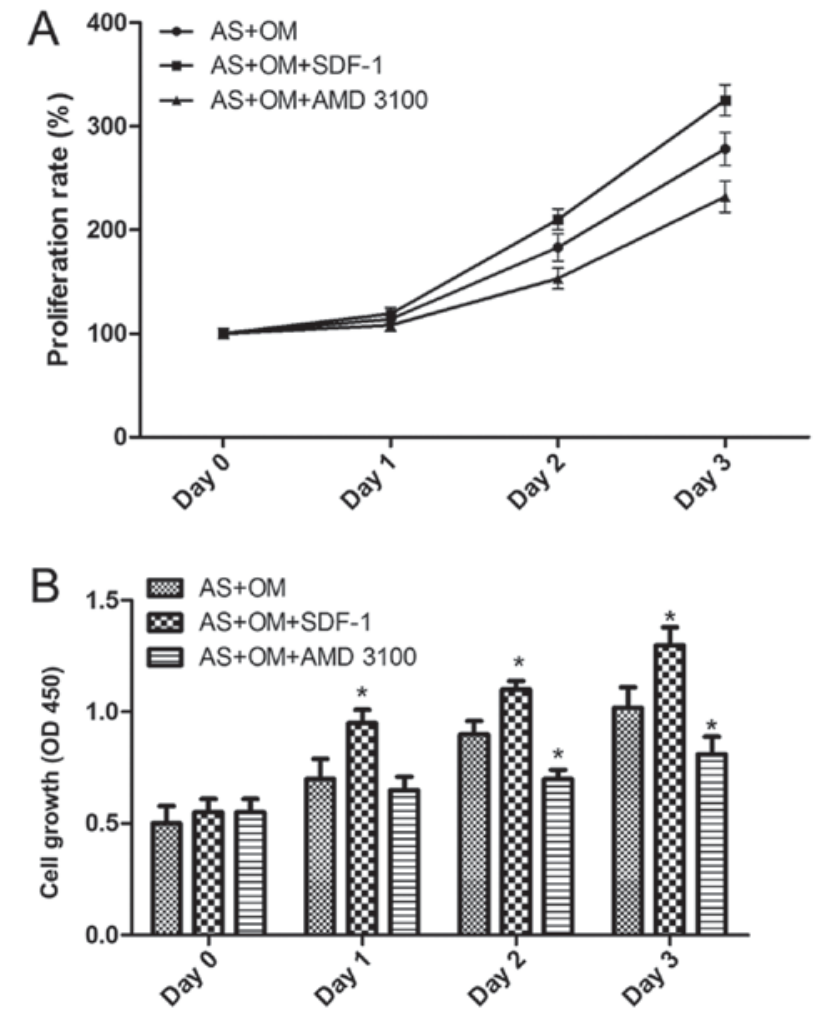

Figure 3. MD 3100 and SDF-1 attenuates AS fibroblasts growth. (A) Blocking CXCR4 suppresses the growth of AS fibroblasts. (B) Examination of cell proliferation by flow cytometric analysis for each group. ${ }^{*} \mathrm{P}<0.05 \mathrm{vs}$. AS+OM. AS, ankylosing spondylitis; OM, osteogenic induction medium; SDF-1, stromal cell-derived factor-1; OD optical density.

CXCR4 may be activated through the Wnt/ $\beta$-catenin signaling pathway.

Increased growth rate and proliferation have been observed in AS fibroblasts (35), which is very similar to the results of the present study, indicating that ossification in AS is associated not only with fibroblast osteogenesis but also with proliferation compared with the controls. Therefore, the inhibition of proliferation of fibroblasts may be a potential strategy to prevent new bone formation in AS. In the present study, inhibition of CXCR4 by AMD 3100 significantly inhibited the proliferation in AS fibroblasts.

Wnt signaling, which is involved in the differentiation of osteoblasts, has been indicated to direct novel bone formation in inflammatory arthritis (36-38). Previous studies have demonstrated that the $\mathrm{Wnt} / \beta$-catenin signaling pathway serves an important role in hetero-ossification (39). In the present study, the expression of $\beta$-catenin-dependent transcription effectors was investigated, and the results provided evidence of an association between the expression of CXCR4 and changes to $\mathrm{Wnt} / \beta$-catenin targets. In addition, the expression of downstream effectors of the Wnt signaling pathway, including cyclin D1 and c-myc, was downregulated following the inhibition of CXCR4, suggesting that the inhibition of CXCR4 may inhibit the proliferation of AS fibroblasts by targeting c-myc and cyclin D1.

Other osteogenesis markers, including ALP and OCN, were also examined. ALP and OCN are classic osteogenesis markers that are highly expressed in ossified tissues $(40,41)$, 


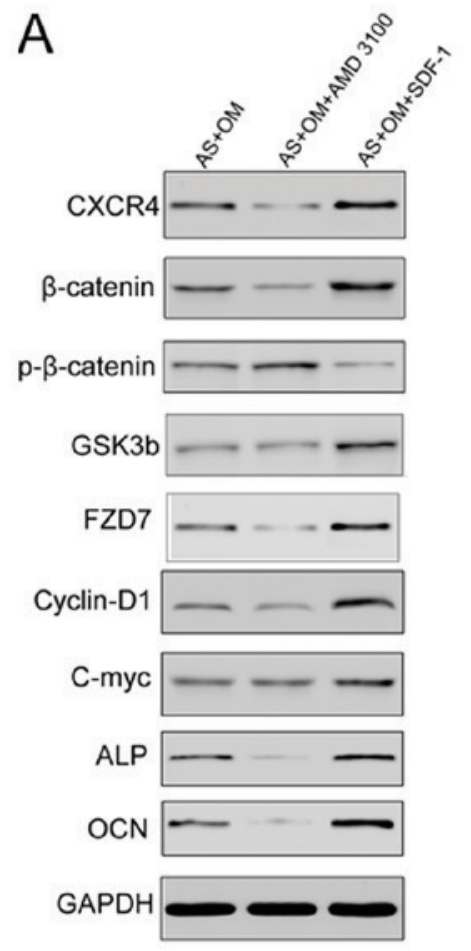

B

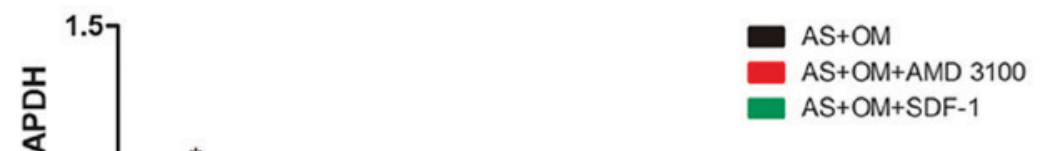

Figure 4. Western blot analysis of the expression levels of $\beta$-catenin, p- $\beta$-catenin, c-myc, cyclin D1, GSK3b, FZD7, ALP and OCN following the addition of AMD 3100/SDF-1 and osteogenesis induction. (A) The data represent the mean value from three independent experiments with 10 samples each. (B) Bar graph demonstrates the results of quantification using Image J software. " $\mathrm{P}<0.05 \mathrm{vs}$. AS+OM. AS, ankylosing spondylitis; OM, osteogenic induction medium; SDF-1, stromal cell-derived factor-1; CXCR4, C-X-C chemokine receptor type 4; p, phosphorylated; GSK3b, glycogen synthase kinase 3b; FZD7, frizzled-7; c-myc, $\mathrm{v}$-myc avian myelocytomatosis viral oncogene homolog; ALP, alkaline phosphatase; OCN, osteocalcin.
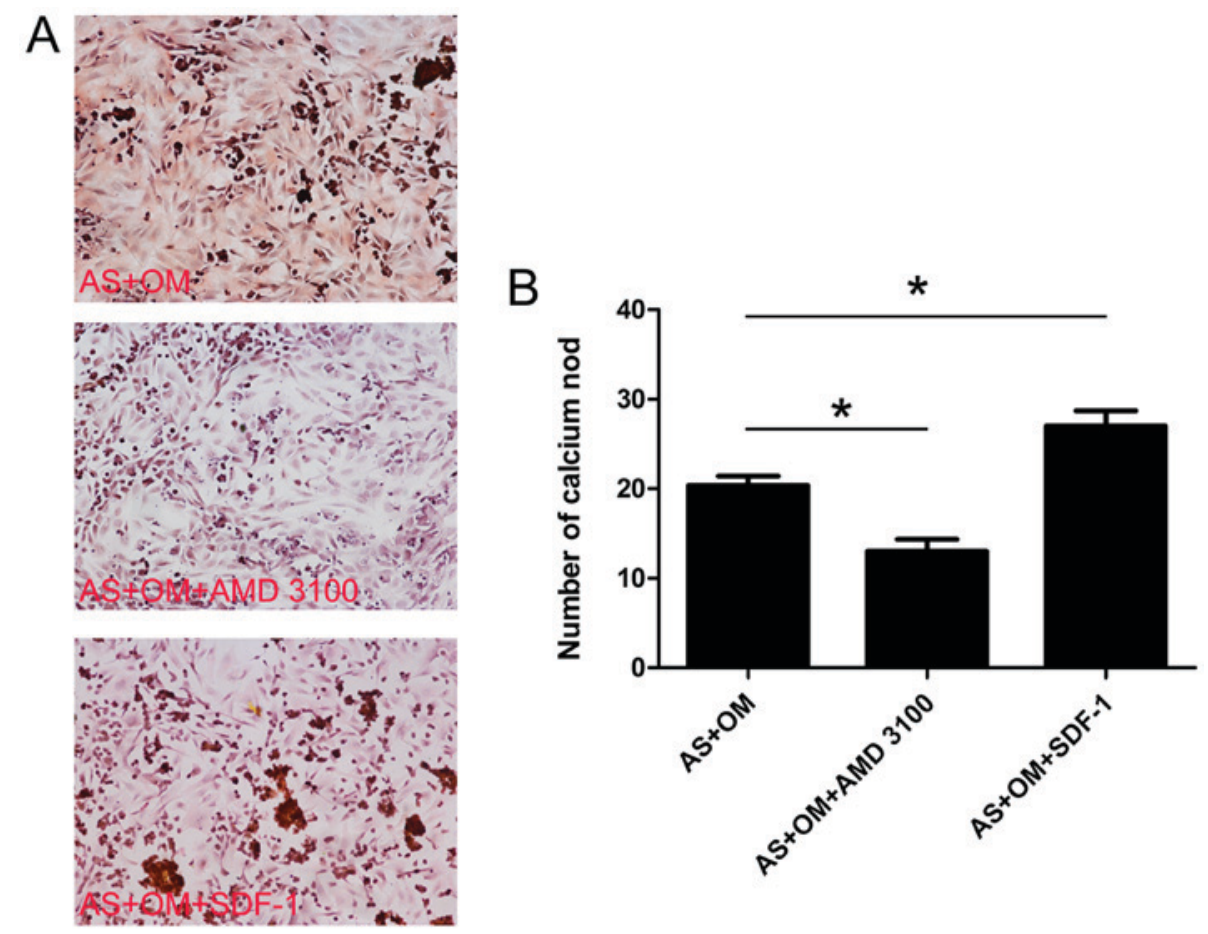

Figure 5. Formation of calcification nodules in AS fibroblasts in each group. (A) Representative image of Alizarin red staining (magnification, $\mathrm{x} 400$ ). (B) Quantification of ossification activity. " $\mathrm{P}<0.05$. AS, ankylosing spondylitis; OM, osteogenic induction medium; SDF-1, stromal cell-derived factor-1.

which may be attenuated by CXCR4 downregulation. To additionally assess osteogenic activity, Alizarin red staining was performed to detect calcium. Decreased mineralization level in the AMD 3100 group was observed. By contrast, the amount of mineralization demonstrated a marked increasing trend in the SDF-1 group, suggesting that upregulated CXCR4 

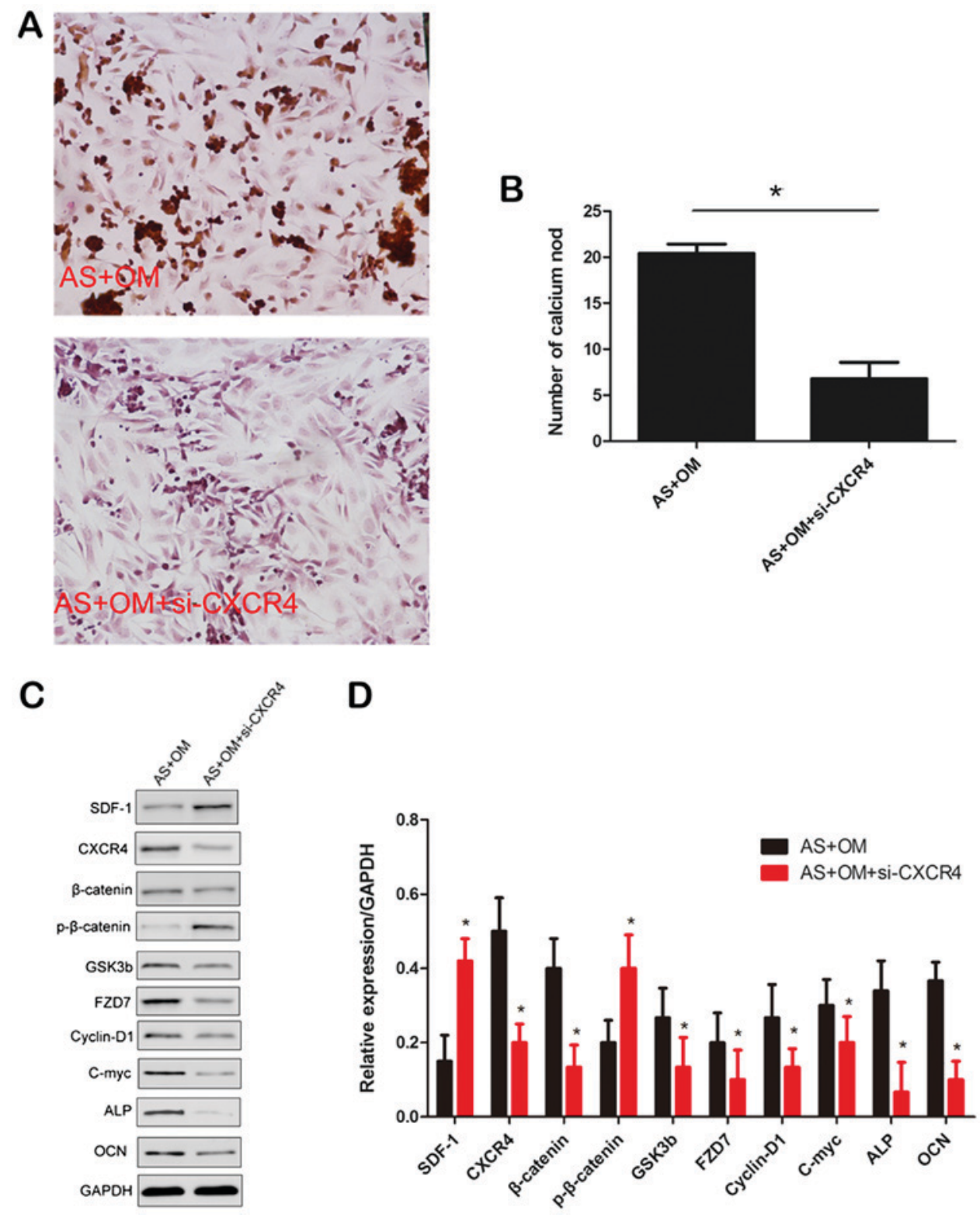

Figure 6. Effects of CXCR4 on osteogenic and mineralization of fibroblasts. (A) Representative images of mineralization formation (magnification, $\mathrm{x} 400$ ). (B) Quantification of mineralization formation level. "P<0.05. (C) Western blot analysis of the expression levels of $\beta$-catenin, $p-\beta$-catenin, $c-M y c$, GSK3b, FZD7, cyclin D1, ALP and OCN following the silencing if CXCR4. (D) Densitometric analysis of the western blot analysis data. Data are presented as mean \pm standard deviation of three independent experiments. "P $<0.05$ vs. AS+OM. AS, ankylosing spondylitis; OM, osteogenic induction medium; si, small interfering RNA; SDF-1, stromal cell-derived factor-1; CXCR4, C-X-C chemokine receptor type 4; p, phosphorylated; GSK3b, glycogen synthase kinase 3b; FZD7, frizzled-7; c-myc, v-myc avian myelocytomatosis viral oncogene homolog; ALP, alkaline phosphatase; OCN, osteocalcin.

increased the level of mineralization in fibroblasts. However, AMD 3100 also decreased mineralization in the normal group, suggesting that additional studies are required for the inhibition of CXCR4 during AS treatment.

In summary, to the best of our knowledge, the present study is the first to have cultured AS fibroblasts and explored the role of CXCR4 in AS hip tissues. It was demonstrated that downregulated CXCR4 levels inhibited fibroblast proliferation and osteogenesis via the Wnt/ $\beta$-catenin signaling pathways in vitro. Therefore, we hypothesize that CXCR4 may serve an important role in AS patient bone formation, which agrees with the conclusions from previous studies (42). Obtaining an improved understanding of the functions of CXCR4 in AS may provide novel insights of how downregulating the Wnt signaling pathway may prevent or delay new bone formation in AS. It should be noted that in the present study, only 12 patients with AS were included, and only in vitro experiments was performed to reveal the function of CXCR4. Therefore, additional in vitro and in vivo experiments are required to confirm the role of CXCR4 in AS.

\section{Acknowledgements}

Not applicable.

\section{Funding}

The present study was funding by Innovation project fund of the Second Military Medical University (grant no. 2017QN04) and 
the National Nature Science Foundation of China (grant nos 81672126 and 81871751).

\section{Availability of data and materials}

All data generated or analyzed during this study are included in this published article.

\section{Authors' contributions}

$\mathrm{CH}$ and DL contributed to experimental design, manuscript writing and experimental techniques. JG performed specimen collection and experimental techniques. JL performed specimen collection and provided experimental assistance. ZL and WX contributed to experimental design.

\section{Ethics approval and consent to participate}

The study was approved by the Ethics Committee of Second Military Medical University. All patients provided written informed consent.

\section{Patient consent for publication}

All patients provided written informed consent.

\section{Competing interests}

The authors declare that they have no competing interest.

\section{References}

1. Sochart DH and Porter ML: Long-term results of total hip replacement in young patients who had ankylosing spondylitis. Eighteen to thirty-year results with survivorship analysis. J Bone Joint Surg Am 79: 1181-1189, 1997.

2. Wordsworth BP and Mowat AG: A review of 100 patients with ankylosing spondylitis with particular reference to socio-economic effects. Br J Rheumatol 25: 175-180, 1986.

3. Tang WM and Chiu KY: Primary total hip arthroplasty in patients with ankylosing spondylitis. J Arthroplasty 15: 52-58, 2000.

4. Brinker MR, Rosenberg AG, Kull L and Cox DD: Primary noncemented total hip arthroplasty in patients with ankylosing spondylitis. Clinical and radiographic results at an average follow-up period of 6 years. J Arthroplasty 11: 802-812, 1996.

5. Vander Cruyssen B, Vastesaeger N and Collantes-Estévez E: Hip disease in ankylosing spondylitis. Curr Opin Rheumatol 25: 448-454, 2013

6. Zhang HY, Liu R, Xing YJ, Xu P, Li Y and Li CJ: Effects of hypoxia on the proliferation, mineralization and ultrastructure of human periodontal ligament fibroblasts in vitro. Exp Ther Med 6: 1553-1559, 2013.

7. Yang HS, Lu XH, Chen DY, Yuan W, Yang LL, He HL and Chen Y: Upregulated expression of connexin43 in spinal ligament fibroblasts derived from patients presenting ossification of the posterior longitudinal ligament. Spine (Phila Pa 1976) 36: 2267-2274, 2011.

8. Sommar P, Junker JP, Strandenes E, Ness C, Hansson T, Johnson $\mathrm{H}$ and Kratz G: Osteogenically-induced human dermal fibroblasts as a tool to regenerate bone. J Plast Surg Hand Surg 47: 8-13, 2013

9. Yu F, Cui Y, Zhou X, Zhang X and Han J: Osteogenic differentiation of human ligament fibroblasts induced by conditioned medium of osteoclast-like cells. Biosci Trends 5: 46-51, 2011.

10. Jones GN, Moschidou D, Lay K, Abdulrazzak H, Vanleene M, Shefelbine SJ, Polak J, de Coppi P, Fisk NM and Guillot PV: Upregulating CXCR4 in human fetal mesenchymal stem cells enhances engraftment and bone mechanics in a mouse model of osteogenesis imperfecta. Stem Cells Transl Med 1: 70-78, 2012.
11. Wang Y, Fu W, Zhang S, He X, Liu Z, Gao D and Xu T: CXCR-7 receptor promotes $S D F-1 \alpha$-induced migration of bone marrow mesenchymal stem cells in the transient cerebral ischemia/reperfusion rat hippocampus. Brain Res 1575: 78-86, 2014.

12. Kioi M, Vogel H, Schultz G, Hoffman RM, Harsh GR and Brown JM: Inhibition of vasculogenesis, but not angiogenesis, prevents the recurrence of glioblastoma after irradiation in mice. J Clin Invest 120: 694-705, 2010.

13. Clift IC, Bamidele AO, Rodriguez-Ramirez C, Kremer KN and Hedin KE: $\beta$-Arrestin1 and distinct CXCR4 structures are required for stromal derived factor- 1 to downregulate CXCR4 cell-surface levels in neuroblastoma. Mol Pharmacol 85: 542-552, 2014.

14. Liu X, Duan B, Cheng Z, Jia X, Mao L, Fu H, Che Y, Ou L, Liu L and Kong D: SDF-1/CXCR4 axis modulates bone marrow mesenchymal stem cell apoptosis, migration and cytokine secretion. Protein Cell 2: 845-854, 2011.

15. Cencioni C, Capogrossi MC and Napolitano M: The SDF-1/CXCR4 axis in stem cell preconditioning. Cardiovasc Res 94: 400-407, 2012.

16. Deng QJ, Xu XF and Ren J: Effects of SDF-1/CXCR4 on the repair of traumatic brain injury in rats by mediating bone marrow derived mesenchymal stem cells. Cell Mol Neurobiol 38: 467-477, 2018.

17. Shahnazari M, Chu V, Wronski TJ, Nissenson RA and Halloran BP: CXCL12/CXCR4 signaling in the osteoblast regulates the mesenchymal stem cell and osteoclast lineage populations. FASEB J 27: 3505-3513, 2013.

18. Kitaori T, Ito H, Schwarz EM, Tsutsumi R, Yoshitomi H, Oishi S, Nakano M, Fujii N, Nagasawa T and Nakamura T: Stromal cell-derived factor 1/CXCR4 signaling is critical for the recruitment of mesenchymal stem cells to the fracture site during skeletal repair in a mouse model. Arthritis Rheum 60: 813-823, 2009.

19. van der Linden S, Valkenburg HA and Cats A: Evaluation of diagnostic criteria for ankylosing spondylitis. A proposal for modification of the New York criteria. Arthritis Rheum 27: 361-368, 1984.

20. Kisselbach L, Merges M, Bossie A and Boyd A: CD90 expression on human primary cells and elimination of contaminating fibroblasts from cell cultures. Cytotechnology 59: 31-44, 2009.

21. Sack U, Hirth A, Funke B, Wiedemeyer K, Lange F, Tröltzsch M, Tannapfel A, Gebhardt R, Emmrich F and Lehmann J: A novel model of fibroblast-mediated cartilage destruction. Scand J Immunol 61: 18-28, 2005.

22. Xu WD, Yang XY, Li DH, Zheng KD, Qiu PC, Zhang W, Li CY, Lei KF, Yan GQ, Jin SW and Wang JG: Up-regulation of fatty acid oxidation in the ligament as a contributing factor of ankylosing spondylitis: A comparative proteomic study. J Proteomics 113: 57-72, 2015

23. Gregory CA, Gunn WG, Peister A and Prockop DJ: An Alizarin red-based assay of mineralization by adherent cells in culture: comparison with cetylpyridinium chloride extraction. Anal Biochem 329: 77-84, 2004.

24. Lories RJ, Derese I, Ceuppens JL and Luyten FP: Bone morphogenetic proteins 2 and 6, expressed in arthritic synovium, are regulated by proinflammatory cytokines and differentially modulate fibroblast-like synoviocyte apoptosis. Arthritis Rheum 48: 2807-2818, 2003.

25. Maksymowych WP, Landewe R, Conner-Spady B, Dougados M, Mielants $H$, van der Tempel $H$, Poole AR, Wang $N$ and van der Heijde D: Serum matrix metalloproteinase 3 is an independent predictor of structural damage progression in patients with ankylosing spondylitis. Arthritis Rheum 56: 1846-1853, 2007.

26. Cai L, Zhang J, Qian J, Li Q, Li H, Yan Y, Wei S, Wei J and $\mathrm{Su}$ J: The effects of surface bioactivity and sustained-release of genistein from a mesoporous magnesium-calcium-silicate/PK composite stimulating cell responses in vitro, and promoting osteogenesis and enhancing osseointegration in vivo. Biomater Sci 6: 842-853, 2018.

27. Marciano DP, Kuruvilla DS, Boregowda SV, Asteian A, Hughes TS, Garcia-Ordonez R, Corzo CA, Khan TM, Novick SJ, Park H, et al: Pharmacological repression of PPAR $\gamma$ promotes osteogenesis. Nat Commun 6: 7443, 2015.

28. Fukada S, Yamamoto Y, Segawa M, Sakamoto K, Nakajima M, Sato M, Morikawa D, Uezumi A, Miyagoe-Suzuki Y, Takeda S, et al: CD90-positive cells, an additional cell population, produce laminin alpha 2 upon transplantation to $\mathrm{dy}(3 \mathrm{k}) / \mathrm{dy}(3 \mathrm{k})$ mice. Exp Cell Res 314: 193-203, 2008. 
29. Dakhova O, Ozen M, Creighton CJ, Li R, Ayala G, Rowley D and Ittmann M: Global gene expression analysis of reactive stroma in prostate cancer. Clin Cancer Res 15: 3979-3989, 2009.

30. Andrade PZ, da Silva CL, dos Santos F, Almeida-Porada G and Cabral JM: Initial CD34+ cell-enrichment of cord blood determines hematopoietic stem/progenitor cell yield upon ex vivo expansion. J Cell Biochem 112: 1822-1831, 2011.

31. Kim B, Lee B, Kim MK, Gong SP, Park NH, Chung HH, Kim HS No JH, Park WY, Park AK, et al: Gene expression profiles of human subcutaneous and visceral adipose-derived stem cells. Cell Biochem Funct 34: 563-571, 2016.

32. Nim HT, Furtado MB, Costa MW, Kitano H, Rosenthal NA and Boyd SE: CARFMAP: A curated pathway map of cardiac fibroblasts. PLoS One 10: e0143274, 2015.

33. Kumar S and Ponnazhagan S: Mobilization of bone marrow mesenchymal stem cells in vivo augments bone healing in a mouse model of segmental bone defect. Bone 50: 1012-1018, 2012.

34. Huang J, Yin Z, Song G, Cui S, Jiang J and Zhang L: Discriminating value of calprotectin in disease activity and progression of nonradiographic axial spondyloarthritis and ankylosing spondylitis. Dis Markers 2017: 7574147, 2017.

35. Zou YC, Yang XW, Yuan SG, Zhang P, Ye YL and Li YK: Downregulation of dickkopf-1 enhances the proliferation and osteogenic potential of fibroblasts isolated from ankylosing spondylitis patients via the $\mathrm{Wnt} / \beta$-catenin signaling pathway in vitro. Connect Tissue Res 57: 200-211, 2016.
36. Kitagaki J, Iwamoto M, Liu JG, Tamamura Y, Pacifci $M$ and Enomoto-Iwamoto M: Activation of beta-catenin-LEF/TCF signal pathway in chondrocytes stimulates ectopic endochondral ossification. Osteoarthritis Cartilage 11: 36-43, 2003.

37. Wendling D and Claudepierre P: New bone formation in axial spondyloarthritis. Joint Bone Spine 80: 454-458, 2013.

38. Wang Y, Li YP, Paulson C, Shao JZ, Zhang X, Wu M and Chen W: Wnt and the Wnt signaling pathway in bone development and disease. Front Biosci (Landmark Ed) 19: 379-407, 2014.

39. Clevers $\mathrm{H}$ and Nusse $\mathrm{R}$ : Wnt $/ \beta$-catenin signaling and disease. Cell 149: 1192-1205, 2012.

40. Li Y, Zheng Z, Cao Z, Zhuang L, Xu Y, Liu X, Xu Y and Gong Y: Enhancing proliferation and osteogenic differentiation of HMSCs on casein/chitosan multilayer films. Colloids Surf B Biointerfaces 141: 397-407, 2016.

41. Zhang L, Tang Y, Zhu X, Tu T, Sui L, Han Q, Yu L, Meng S, Zheng L, Valverde P, et al: Overexpression of MiR-335-5p promotes bone formation and regeneration in mice. J Bone Miner Res 32: 2466-2475, 2017.

42. Dong Y, Liu H, Zhang X, Xu F, Qin L, Cheng P, Huang H, Guo F, Yang $\mathrm{Q}$ and Chen A: Inhibition of SDF- $1 \alpha / \mathrm{CXCR} 4$ signalling in subchondral bone attenuates post-traumatic osteoarthritis. Int J Mol Sci 17: pii: E943, 2016. 